\title{
Modelling of Hybrid Materials and Interface Defects through Homogenization Approach for the Prediction of Effective Thermal Conductivity of FRP Composites Using Finite Element Method
}

\author{
C. Mahesh, ${ }^{1}$ K. Govindarajulu, ${ }^{2}$ and V. Balakrishna Murthy ${ }^{1}$ \\ ${ }^{1}$ Department of Mechanical Engineering, V.R. Siddhartha Engineering College, Vijayawada 520 007, India \\ ${ }^{2}$ Department of Mechanical Engineering, JNT University Anantapur, Anantapur 521 501, India
}

Correspondence should be addressed to C. Mahesh; mahesh_chindanuru@rediffmail.com

Received 30 May 2013; Accepted 12 September 2013

Academic Editor: Martha Guerrero

Copyright (C) 2013 C. Mahesh et al. This is an open access article distributed under the Creative Commons Attribution License, which permits unrestricted use, distribution, and reproduction in any medium, provided the original work is properly cited.

Finite element method is effectively used to homogenize the thermal conductivity of FRP composites consisting of hybrid materials and fibre-matrix debonds at some of the fibres. The homogenized result at microlevel is used to determine the property of the layer using macromechanics principles; thereby, it is possible to minimize the computational efforts required to solve the problem as in state through only micromechanics approach. The working of the proposed procedure is verified for three different problems: (i) hybrid composite having two different fibres in alternate layers, (ii) fibre-matrix interface debond in alternate layers, and (iii) fibrematrix interface debond at one fibre in a group of four fibres in one unit cell. It is observed that the results are in good agreement with those obtained through pure micro-mechanics approach.

\section{Introduction}

Composite materials are extensively used in many fields of engineering such as aerospace, electronic packaging, reactor vessels, and turbines, due to light weight, high strength, long durability, stability against chemical reaction, tailorable properties, and so forth. FRP composites can be designed as heat conductors for enhancing heat transfer rate and also for insulation purpose; this depends on the thermal properties, volume fraction, orientation, and so forth, of each constituent of the composite. The effective thermal conductivity and other thermophysical properties of composites have been a topic of considerable theoretical, experimental, and numerical interest from long period.

Composite materials are nonhomogeneous and exhibit anisotropic response due to structural and thermal loads. Analysis of a composite structure as in state of heterogeneity by providing the material properties of constituent materials is mathematically complex, and therefore theories such as micromechanics and macromechanics are developed for the theoretical analysis. The homogenized properties of a composite lamina obtained from micromechanical theories are used for the macromechanical analysis of a composite made of several individual laminas stacked in a specified manner.

The micromechanical theories select a particular portion of the composite known as "Representative Volume Elements" (RVE) and find the properties of RVE which are considered to be lamina properties. In this approach, there are many assumptions such as fibres which are arranged in a particular pattern (square/hexagonal) in a matrix, no voids in the matrix, all fibres are of uniform cross-section and perfectly aligned, the interface between the fibre and matrix is perfectly or totally debond; this leads to much deviation of theoretical and experimental results. Numerical approaches such as Finite Element Method (FEM) are developed to overcome some of the assumptions of micromechanical theories but still not explored in addressing many complexities in micromechanical approach. Though FEM is an 
approximate method, it can be effectively used after proper mesh refinement and validation.

Aligned fibre composite laminates are frequently used in beam, plate, or shell form. The axial thermal conductivity (in the fibre direction) of each lamina is satisfactorily predicted by a simple rule of mixtures Chawla [1]. Prediction of through-thickness thermal conductivity is more problematic. Yet this is important, since heat sources one side of the laminate often create a through-thickness temperature gradient.

Earlier several researchers studied thermal conductivities of composites by experimental, theoretical, and numerical approaches. Prediction of effective transverse thermal conductivity of fibre reinforced composites is made for several models, such as experimental determination of effective thermal conductivity of aligned fibre composite of Chamis [2], thermal conductivity of constituents of FRCL by backout method of Al-Sulaiman et al. [3], simple thermal resistance models of Chawla, Springer and Tsai [1, 4], as well as sophisticated conduction models of Rayleigh and $\mathrm{Lu}[5,6]$ and interface resistance models of Hasselman and Johnson, Farmer and Covert, Zou et al., Benveniste [7-10], 2D numerical model of Islam and Pramila, [11], 3D numerical model of Rao et al. [12], 2D thermal contact resistance model of Ramani and Vaidyanathan, [13]. Hybrid composites are also playing a dominant role by adding desirable properties which are lagging with monofibre composites by Jawaid and Abdul Khalil [14].

In the present analysis, a 3D FEM is proposed to address various nonsimilarities in the unit cells at microlevel and developing equivalence between micro- and macromechanical approaches through some of the examples of heat conduction. Three cases are considered for present study: case (i) hybrid composite constituting two different thermal conductivity fibres and matrix, case (ii) composite with alternate layers of fibres fully debonded, and case (iii) composite with one in set of four fibres of a unit cell is fully debonded.

\section{Problem Modelling}

2.1. Geometric Modelling. Figures 1, 3, and 5 represent the microlevel view of case (i) hybrid composite constituting two different thermal conductivity fibres and matrix, case (ii) composite with alternate layers of fibres fully debonded, and case (iii) composite with one in set of four fibres of a unit cell debonded, respectively. Because of high degree of symmetry in the alignment of fibres, thermal loading, one-fourth model is used for the analysis as shown in Figures 2 and 4. For case (iii), even though material and geometric symmetry exists, due to non-symmetric thermal loading a full model is taken for the analysis as shown in Figure 6. Dimensions considered for the analysis are $a=100$ units, $b=100$ units, radius of fibres corresponds to volume fractions ranging from 0.1 to 0.7 .

In macromechanics approach for first two cases, two blocks are modelled one over the other. For case (i) one block represents fibrel and matrix portion and the other block is for fibre 2 and matrix portion. Case (ii) one block represents total debonded fibre matrix and other perfectly bonded fibre matrix, whereas for case (iii) four blocks are modelled in

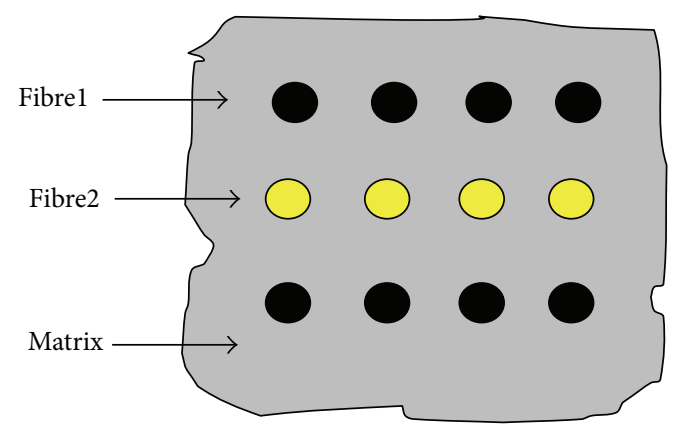

Figure 1: Concept of square unit cell for aligned pattern of hybrid composite with two different types of fibres.

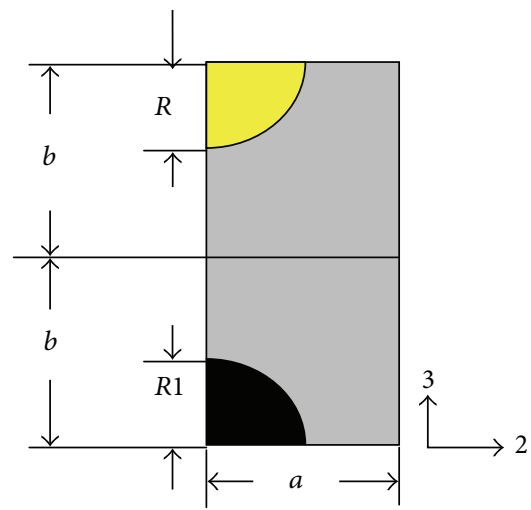

FIGURE 2: One-fourth model of unit cell for aligned pattern of fibres.

square pattern. One block represents debonded fibre and matrix portion and other three blocks for perfectly bonded fibre matrix. Surfaces at the junction of blocks are merged for heat transfer connectivity without any interfacial thermal barrier.

2.2. Finite Element Modelling. The problem is modelled in commercial Finite Element Software ANSYS 12 [15]. A 3D quadratic brick element having 20 nodes with a single degree of freedom (temperature) at each node, named SOLID90, is used for discretization of the constituents. For hybrid composite two material properties are assigned for fibrel and fibre2, whereas for case (ii) and case (iii) only one material property is assigned for fibres. CONTA174 and TARGE170 elements of ANSYS software are used for studying interface thermal contact behaviour between fibre and matrix. The finite element mesh is properly refined, and converged results are verified with Hasselman-Johnson and FarmerCovert models for perfect bond and total debond at fibrematrix interfaces. Results are present in Section 3. Figure 7 represents the Geometry and FE mesh of a 3D model for case (i) and case (ii) and Figure 8 represents the Geometry and FE mesh of a 3D model for case (iii).

2.3. Boundary Conditions. Temperature difference $(d T)$ of $100^{\circ} \mathrm{C}$ is maintained on two isothermal surfaces perpendicular to heat flow direction. All other surfaces are subjected to insulation boundary conditions. For debond between fibre 


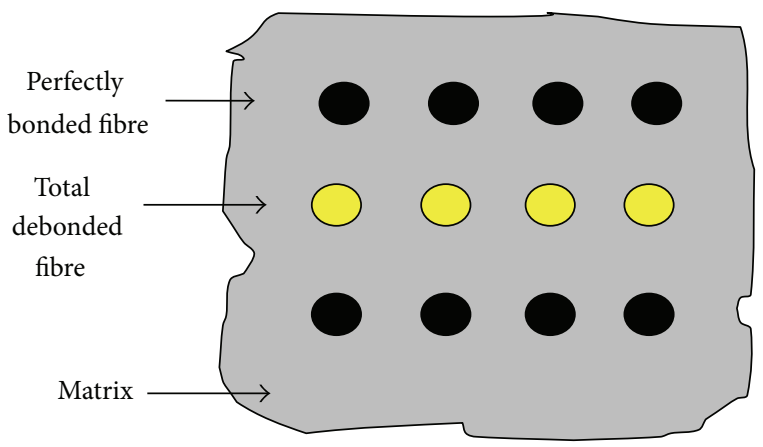

Figure 3: Concept of square unit cell for aligned pattern of composite with alternate layers of debonded fibres.

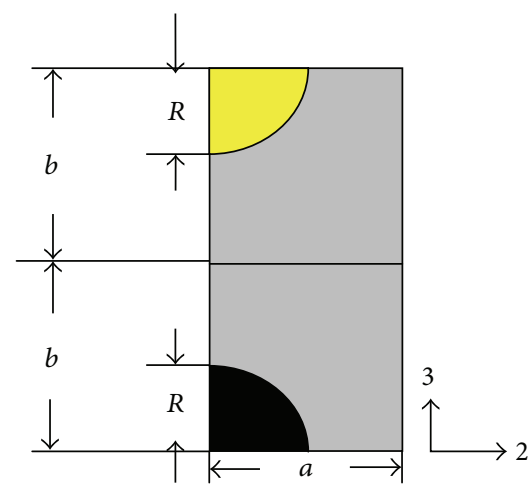

FIGURE 4: One-fourth model of unit cell for composite with alternate layers of debonded fibres.

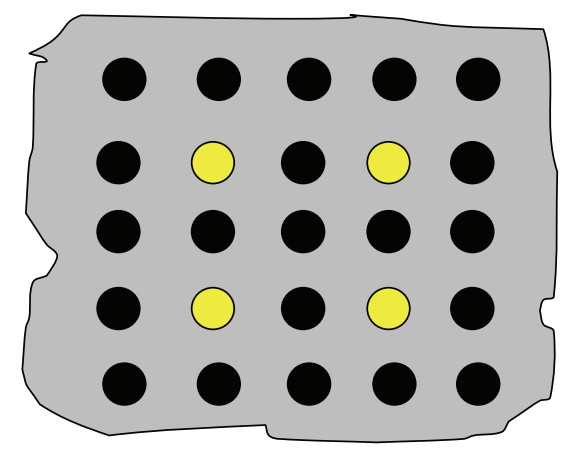

FIGURE 5: Concept of square unit cell for aligned pattern of composite with one in set of four fibres of unit cell totally debonded.

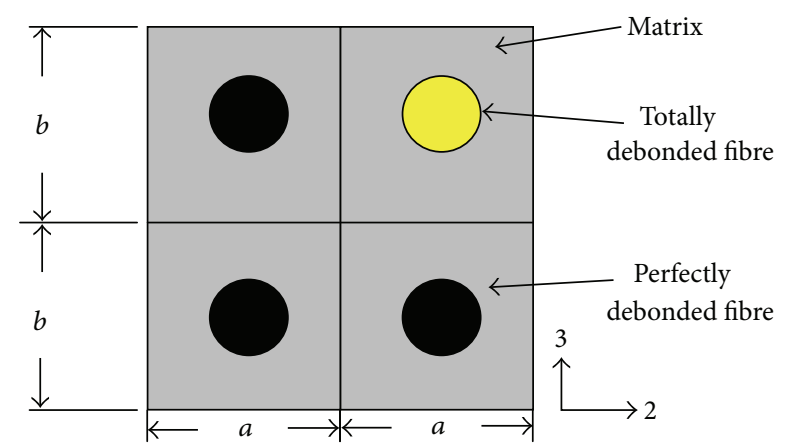

FIGURE 6: Full model for aligned pattern of composite with one in set of four fibres of unit cell totally debonded.

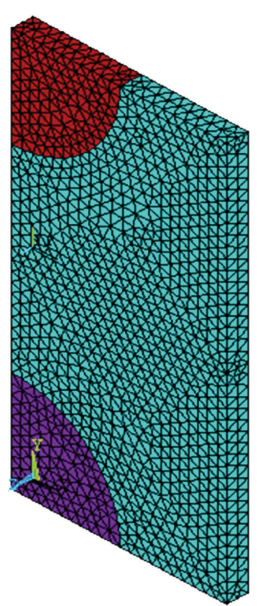

FIGURE 7: Geometry and FE mesh of a 3D model for case (i) and case (ii).

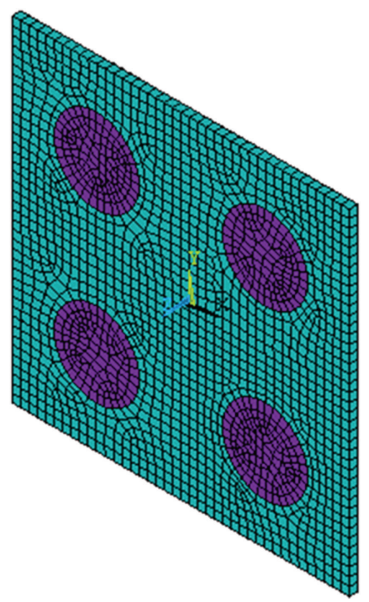

FIGURE 8: Geometry and FE mesh of a 3D model for case (iii).

and matrix contact conductance $h_{c}=1 E-6$ and for perfect contact between fibre and matrix contact conductance $h_{c}=$ 1 E6 are taken.

2.4. Material Properties. For the validation of the models developed in the present analysis, the following properties of fibres and matrix [2] are considered.

Polyimide matrix with thermal conductivity $K_{m}=$ $0.19 \mathrm{~W} / \mathrm{mK}$.

(T-300) Carbon fibre with thermal conductivity $K_{f 1}=$ $8.365218 \mathrm{~W} / \mathrm{mK}$.

For all the three cases of the study, matrix is polyimide matrix.

For the hybrid model, fibrel : fibre $2:$ matrix thermal conductivity ratios used are $100: 10: 1,80: 8: 1,60: 6: 1$, and $40: 4: 1$.

For the debond models, fibre thermal conductivities range from $0.14 \mathrm{~W} / \mathrm{mK}$ to $1 E 6 \mathrm{~W} / \mathrm{mK}$. 


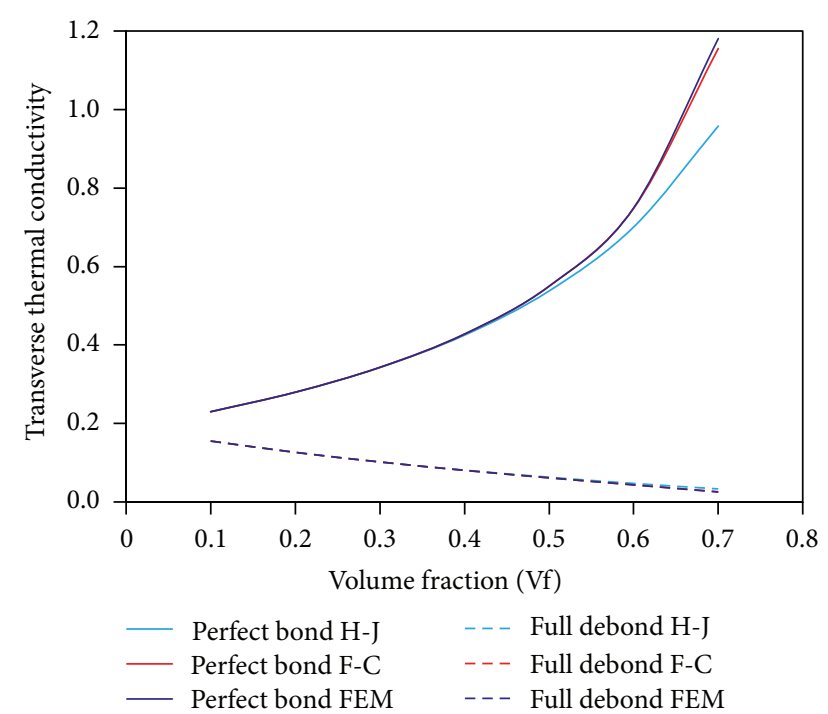

FIGURE 9: Variation in transverse thermal conductivity with respect to Vf for perfect bond and total debond conditions.

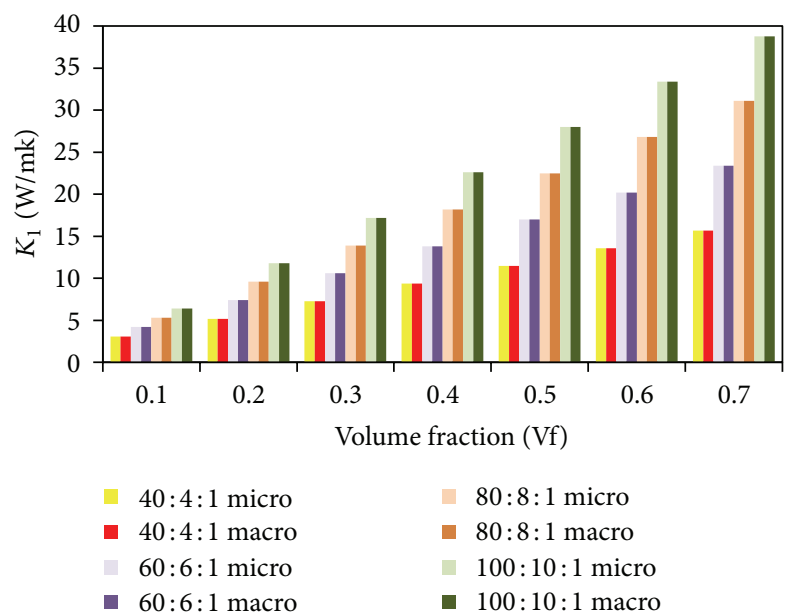

FIGURE 10: Variation in $K_{1}$ of hybrid composite with respect to Vf for different $K_{f 1}: K_{f 2}: K_{m}$.

\section{Discussion of Results}

The analytical solution for 1D heat conduction in homogeneous slabs is readily available. However, the analytical solution for $1 \mathrm{D}$ heat conduction is quite complex for heterogeneous materials such as fibre reinforced composite materials. Thus, the numerical finite element models have been developed to suit the different cases under consideration for this study. The models are first tested for mesh-independent solution by imposing earlier stipulated boundary conditions; then with the heat flux obtained from ANSYS software, effective thermal conductivity of composite is found by Fourier's law of heat conduction [3],

$$
q=-K \frac{d T}{d x}
$$

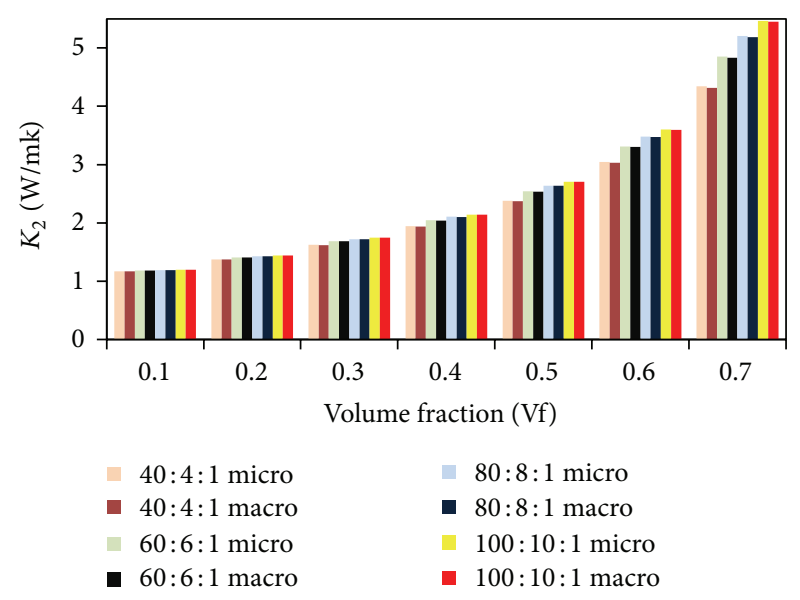

FIGURE 11: Variation in $K_{2}$ of hybrid composite with respect to Vf for different $K_{f 1}: K_{f 2}: K_{m}$.

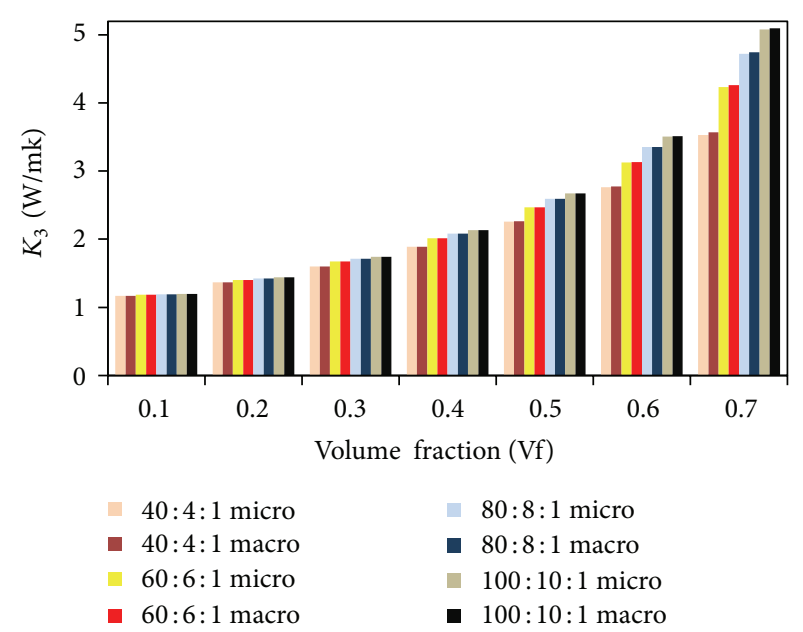

FIGURE 12: Variation in $K_{3}$ with respect to Vf of hybrid composite for different $K_{f 1}: K_{f 2}: K_{m}$.

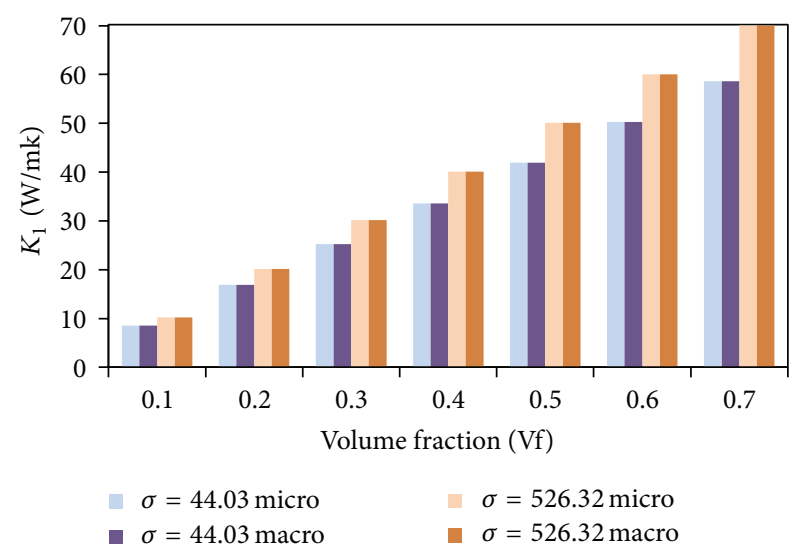

FIGURE 13: Variation in $K_{1}$ with respect to Vf of a composite with alternate layers of fibres fully debonded. 


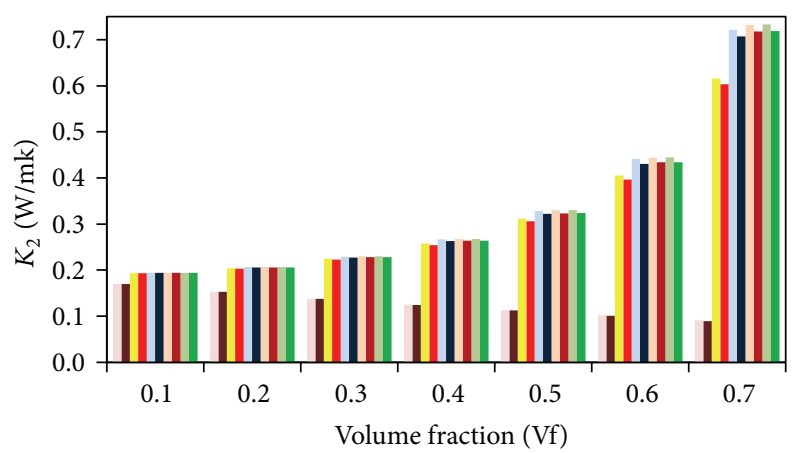

$$
\begin{array}{ll}
\sigma=0.74 \text { micro } & \square=526.32 \text { macro } \\
\sigma=0.74 \text { macro } & \sigma=5263.2 \text { micro } \\
\sigma=44.03 \text { micro } & \square \sigma=5236.2 \text { macro } \\
\sigma=44.03 \text { macro } & \square=\alpha \text { micro } \\
\sigma=526.32 \text { micro } & \square \sigma=\alpha \text { macro }
\end{array}
$$

FIGURE 14: Variation in $K_{2}$ with respect to Vf of a composite with alternate layers of fibres fully debonded.

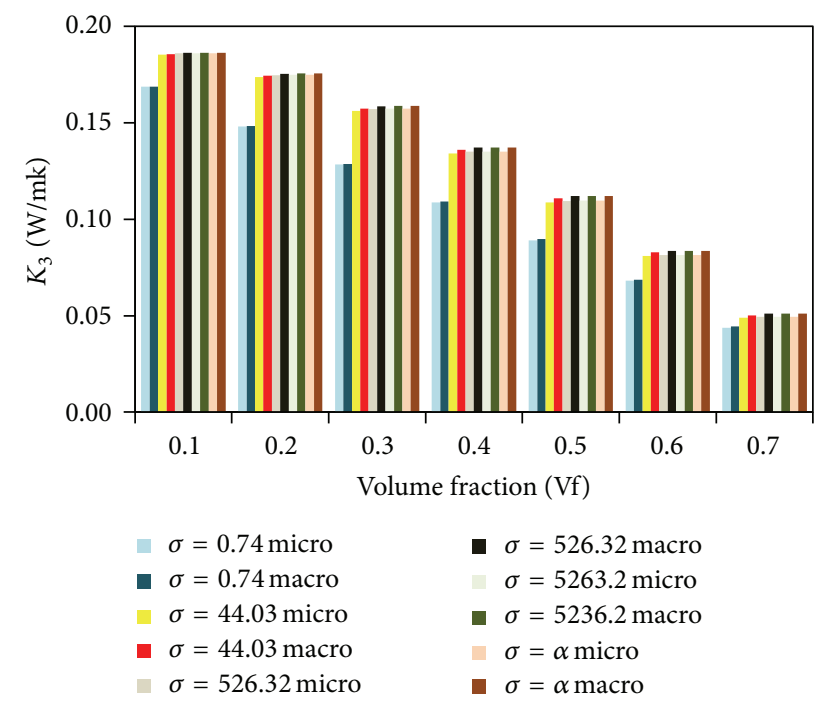

FIGURE 15: Variation in $K_{3}$ with respect to Vf of a composite with alternate layers of fibres fully debonded.

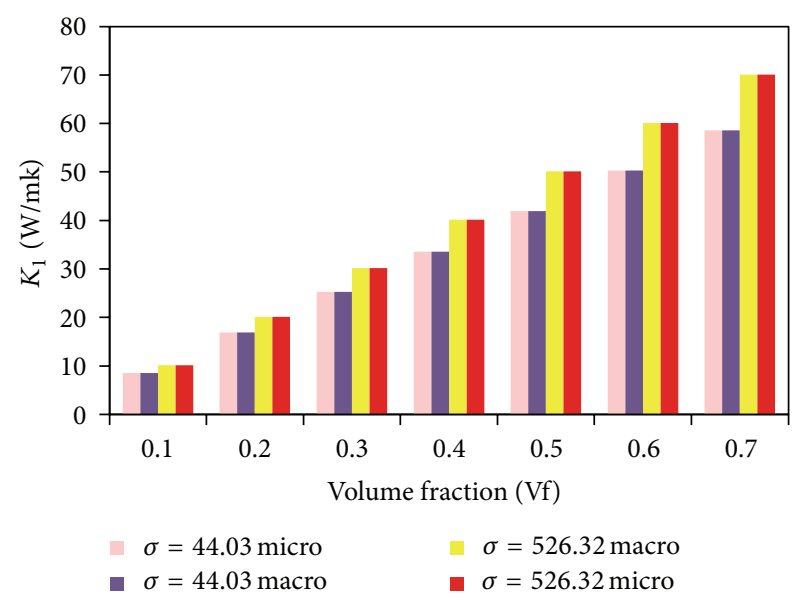

FIgURE 16: Variation in $K_{1}$ with respect to Vf for a composite with one in four fibres in unit cell fully debonded.

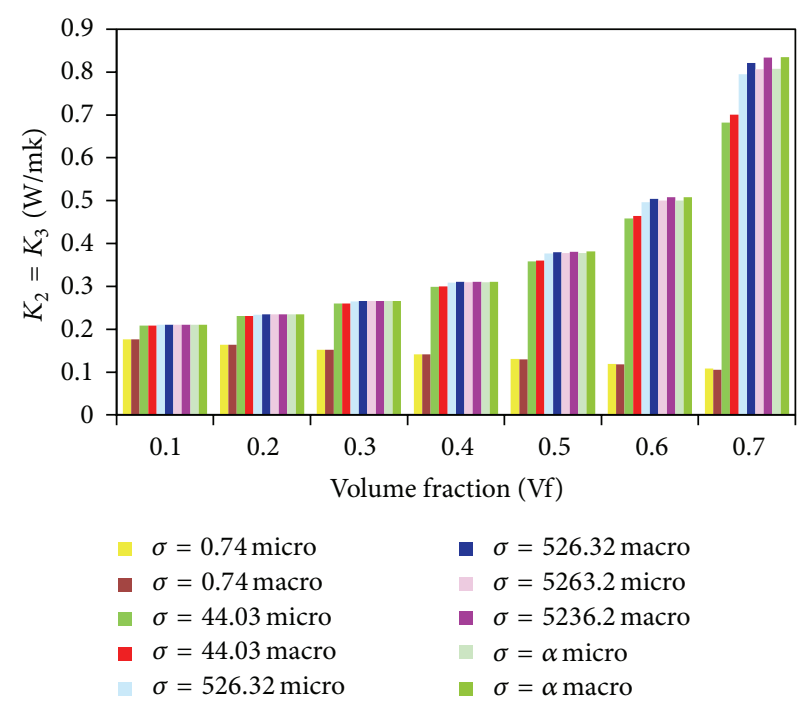

FIgURE 17: Variation of $K_{2}=K_{3}$ with respect to Vf for a composite with one in four fibres in unit cell fully debonded.

where $d T / d x$ is temperature gradient between two isothermal surfaces. $q$ is the heat flux $\mathrm{W} / \mathrm{m}^{2}$.

FE model developed in ANSYS is first validated with the Hasselman and Johnson model (H-J) [7] and Farmer and Covert model (F-C) [8]. Figure 9 shows the comparison of results between Hasselman-Johnson model, Farmer-Covert model, and FEM model. It reveals that the results predicted by FE model for full debond case exactly match for complete range of volume fraction and up to nearly $50 \% \mathrm{Vf}$ for perfect bond condition with both H-J and F-C models and deviation with $\mathrm{H}-\mathrm{J}$ model from $50 \%$ Vf is due to assumptions made in the model and quite coherent with the higher order F-C model.

Figures 10, 11, and 12 show variation in principal thermal conductivities with respect to volume fraction and fibrel: fibre2: matrix thermal conductivity ratios for case (i).

Figures 13, 14, and 15 show variation in principal thermal conductivities with respect to volume fraction and fibrematrix thermal conductivity ratios for case (ii).

Figures 16 and 17 show variation in principal thermal conductivities with respect to volume fraction and fibrematrix thermal conductivity ratios for case (iii).

Table 1 represents \% error between micro- and macromechanics approaches for case (i).

Table 2 represents \% error between micro- and macromechanics approaches for case (ii).

Table 3 represents \% error between micro- and macromechanics approaches for case (iii).

Effective longitudinal thermal conductivity for three cases matches with the simple rule of mixtures and increases in linear manner with the increase in Vf (Figures 10, 13, and 16) since the fibre thermal conductivity dominates the matrix thermal conductivity. $K_{1}$ increases with increase in fibrel: fibre 2: matrix thermal conductivity ratios and its effect is significant at higher Vf. As low and high $K_{f} / K_{m}$ values prevent better pictorial visibility, in Figures 13 and 
TABLE 1: \% Error between macro- and micromechanics approach results for case-(i) hybrid composite.

\begin{tabular}{|c|c|c|c|c|c|c|c|c|}
\hline \multirow{3}{*}{ Vf } & \multicolumn{2}{|c|}{$100: 10: 01$} & \multicolumn{2}{|c|}{$80: 08: 01$} & \multicolumn{2}{|c|}{$60: 06: 01$} & \multicolumn{2}{|c|}{$40: 04: 01$} \\
\hline & $K_{2}$ & $K_{3}$ & $K_{2}$ & $K_{3}$ & $K_{2}$ & $K_{3}$ & $K_{2}$ & $K_{3}$ \\
\hline & \% Error & \% Error & $\%$ Error & \% Error & \% Error & $\%$ Error & \% Error & $\%$ Error \\
\hline 0.1 & 0.025 & 0.022 & 0.008 & 0.010 & 0.000 & 0.003 & 0.000 & 0.010 \\
\hline 0.2 & 0.021 & 0.013 & 0.007 & 0.024 & 0.028 & 0.006 & 0.029 & 0.022 \\
\hline 0.3 & 0.017 & 0.009 & 0.029 & 0.016 & 0.065 & 0.004 & 0.074 & 0.057 \\
\hline 0.4 & 0.037 & 0.028 & 0.057 & 0.038 & 0.093 & 0.060 & 0.160 & 0.095 \\
\hline 0.5 & 0.085 & 0.037 & 0.125 & 0.046 & 0.173 & 0.097 & 0.294 & 0.177 \\
\hline 0.6 & 0.136 & 0.103 & 0.193 & 0.143 & 0.269 & 0.230 & 0.440 & 0.389 \\
\hline 0.7 & 0.212 & 0.369 & 0.273 & 0.502 & 0.371 & 0.708 & 0.521 & 1.092 \\
\hline
\end{tabular}

TABLE 2: \% Error between macro- and micromechanics approaches for composite with alternate layers of fibres fully debonded.

\begin{tabular}{|c|c|c|c|c|c|c|c|c|c|c|}
\hline \multirow{3}{*}{ Vf } & \multicolumn{2}{|c|}{$K_{f} / K_{m}=0.74$} & \multicolumn{2}{|c|}{$K_{f} / K_{m}=4.03$} & \multicolumn{2}{|c|}{$K_{f} / K_{m}=526.32$} & \multicolumn{2}{|c|}{$K_{f} / K_{m}=5263.2$} & \multicolumn{2}{|c|}{$K_{f} / K_{m}=\alpha$} \\
\hline & $K_{2}$ & $K_{3}$ & $K_{2}$ & $K_{3}$ & $K_{2}$ & $K_{3}$ & $K_{2}$ & $K_{3}$ & $K_{2}$ & $K_{3}$ \\
\hline & $\%$ Error & $\%$ Error & $\%$ Error & $\%$ Error & $\%$ Error & $\%$ Error & $\%$ Error & $\%$ Error & $\%$ Error & \% Error \\
\hline 0.1 & 0.01 & 0.02 & 0.10 & 0.10 & 0.07 & 0.12 & 0.09 & 0.11 & 0.09 & 0.10 \\
\hline 0.2 & 0.01 & 0.13 & 0.37 & 0.34 & 0.35 & 0.43 & 0.35 & 0.43 & 0.34 & 0.44 \\
\hline 0.3 & 0.06 & 0.26 & 0.82 & 0.77 & 0.82 & 0.88 & 0.82 & 0.88 & 0.82 & 0.88 \\
\hline 0.4 & 0.20 & 0.41 & 1.37 & 1.34 & 1.38 & 1.51 & 1.39 & 1.51 & 1.40 & 1.51 \\
\hline 0.5 & 0.31 & 0.88 & 1.89 & 1.93 & 1.92 & 2.27 & 1.93 & 2.28 & 1.94 & 2.28 \\
\hline 0.6 & 0.75 & 0.87 & 2.24 & 2.30 & 2.28 & 2.53 & 2.30 & 2.54 & 2.30 & 2.54 \\
\hline 0.7 & 1.45 & 1.82 & 2.03 & 2.08 & 1.97 & 3.08 & 1.96 & 3.09 & 1.97 & 3.09 \\
\hline
\end{tabular}

TABLE 3: \% Error between macro- and micromechanics approach results for composite with one out of four fibres of unit cell fully debonded.

\begin{tabular}{|c|c|c|c|c|c|}
\hline Vf & $\begin{array}{c}K_{f} / K_{m}=0.74 \\
K_{2}=K_{3} \\
\% \text { Error }\end{array}$ & $\begin{array}{c}K_{f} / K_{m}=44.03 \\
K_{2}=K_{3} \\
\% \text { Error }\end{array}$ & $\begin{array}{c}K_{f} / K_{m}=526.32 \\
K_{2}=K_{3} \\
\% \text { Error }\end{array}$ & $\begin{array}{c}K_{f} / K_{m}=5263.2 \\
K_{2}=K_{3} \\
\% \text { Error }\end{array}$ & $\begin{array}{c}K_{f} / K_{m}=\alpha \\
K_{2}=K_{3} \\
\% \text { Error }\end{array}$ \\
\hline 0.1 & 0.01 & 0.01 & 0.03 & 0.01 & 0.01 \\
\hline 0.2 & 0.01 & 0.02 & 0.06 & 0.06 & 0.08 \\
\hline 0.3 & 0.03 & 0.10 & 0.15 & 0.15 & 0.15 \\
\hline 0.4 & 0.15 & 0.26 & 0.36 & 0.37 & 0.35 \\
\hline 0.5 & 0.34 & 0.61 & 0.79 & 0.79 & 0.78 \\
\hline 0.6 & 0.93 & 1.27 & 1.54 & 1.56 & 1.56 \\
\hline 0.7 & 2.08 & 2.65 & 3.23 & 3.28 & 3.28 \\
\hline
\end{tabular}

16 plots for $K_{f} / K_{m}$ values of 44.03 and 526.32 are made, as the focus of this paper is comparison between micro- and macromechanics approaches. Also for the three cases, $\%$ error between micro- and macromechanics results is zero, so \% error for effective longitudinal thermal conductivity is not shown in tables given below.

Transverse thermal conductivities $K_{2}$ and $K_{3}$ for case (i) vary nonlinearly with the variation in $\mathrm{Vf}$, at low values of Vf a gradual rise in $K_{2}$ and $K_{3}$ and at higher Vf beyond 0.6 a steep rise in $K_{2}$ and $K_{3}$ is observed (Figures 11 and 12). Fibrel: fibre 2 : matrix thermal conductivity ratio is not significant at lower Vf and an increasing trend with respect to $K_{f} / K_{m}$ is observed at higher Vf.\% error between microand macromechanics approaches for $K_{2}$ and $K_{3}$ lies between 0.01 and 1.09 (Table 1).
For case (ii) inplane thermal conductivity $K_{2}$ and case (iii) both transverse thermal conductivities $K_{2}$ and $K_{3}$ vary nonlinearly with volume fraction, and behaviour is similar to case (i) for $K_{f} / K_{m}$ values greater than unity, but these properties decrease linearly with increase in Vf for $K_{f} / K_{m}$ value less than unity. Thermal conductivity mismatch ratio is significant at higher volume fractions. \% error between micro- and macromechanics approaches for $K_{2}$ lies between 0.01 and 3.09 (Table 2).

For case (ii) through-thickness thermal conductivity $K_{3}$ decreases almost linearly with increase in $\mathrm{Vf}$, and there is no significant effect of thermal conductivity mismatch ratio at all volume fractions. \% error between micro- and macromechanics approaches for $K_{2}$ lies between 0.01 and 3.28 (Table 3). 


\section{Conclusions}

Applicability of homogenization technique by using finite element method is successfully tested for predicting effective thermal conductivities of a hybrid and thermal contact resistance models for different volume fractions within practically possible range of $10 \%$ to $70 \%$ and thermal conductivity mismatch ratio 0.74 to $\alpha$. It is evident from the results that macro-mechanics approach yields minimal errors with minimum effort. The same can be extended to composite with more other kinds of dissimilarities which is quite difficult with pure micromechanical models.

\section{References}

[1] K. K. Chawla, Composite Materials, Science and Engineering, Springer, New York, NY, USA, 1987.

[2] C. C. Chamis, "Simplified composite micromechanics equations for hygral, thermal and mechanical properties," NASA Technical Memorandum 83320, 1983.

[3] F. A. Al-Sulaiman, Y. N. Al-Nassar, and E. M. A. Mokheimer, "Prediction of the thermal conductivity of the constituents of fiber-reinforced composite laminates: voids effect," Journal of Composite Materials, vol. 40, no. 9, pp. 797-814, 2006.

[4] G. S. Springer and S. W. Tsai, "Thermal conductivities of unidirectional materials," Journal of Composite Materials, vol. 1, pp. 166-173, 1967.

[5] L. Rayleigh, "On the influence of obstacles arranged in rectangular order upon the properties of a medium," Philosophical Magazine, vol. 34, p. 481, 1982.

[6] S.-Y. Lu, "Effective thermal conductivities of composites with 2D arrays of circular and square cylinders," Journal of Composite Materials, vol. 29, no. 4, pp. 483-506, 1995.

[7] D. P. H. Hasselman and L. F. Johnson, "Effective thermal conductivity of composites with interfacial thermal barrier resistance," Journal of Composite Materials, vol. 21, no. 6, pp. 508-515, 1987.

[8] J. D. Farmer and E. E. Covert, "Transverse thermal conductance of thermosetting composite materials during their cure," in Proceedings of the 34th AIAA/ASME/ASCE/AHS/ASC Structures, Structural Dynamics, and Materials Conference, pp. 2337-2347, April 1993, paper 93.

[9] M. Zou, B. Yu, and D. Zhang, "An analytical solution for transverse thermal conductivities of unidirectional fibre composites with thermal barrier," Journal of Physics D, vol. 35, no. 15, pp. 1867-1874, 2002.

[10] Y. Benveniste, "Effective thermal conductivity of composites with a thermal contact resistance between the constituents: nondilute case," Journal of Applied Physics, vol. 61, no. 8, pp. 2840-2843, 1987.

[11] R. Islam and A. Pramila, "Thermal conductivity of fiber reinforced composites by the FEM," Journal of Composite Materials, vol. 33, no. 18, pp. 1699-1715, 1999.

[12] G. S. Rao, T. Subramanyam, and V. B. Murthy, “3-D finite element models for the prediction of effective transverse thermal conductivity of unidirectional fibre reinforced composites," International Journal of Applied Engineering Research, vol. 3, no. 1, pp. 99-108, 2008.

[13] K. Ramani and A. Vaidyanathan, "Finite element analysis of effective thermal conductivity of filled polymeric composites,"
Journal of Composite Materials, vol. 29, no. 13, pp. 1725-1740, 1995.

[14] M. Jawaid and H. P. S. Abdul Khalil, "Cellulosic/synthetic fibre reinforced polymer hybrid composites: a review," Carbohydrate Polymers, vol. 86, no. 1, pp. 1-18, 2011.

[15] ANSYS Reference Manuals, 2011. 

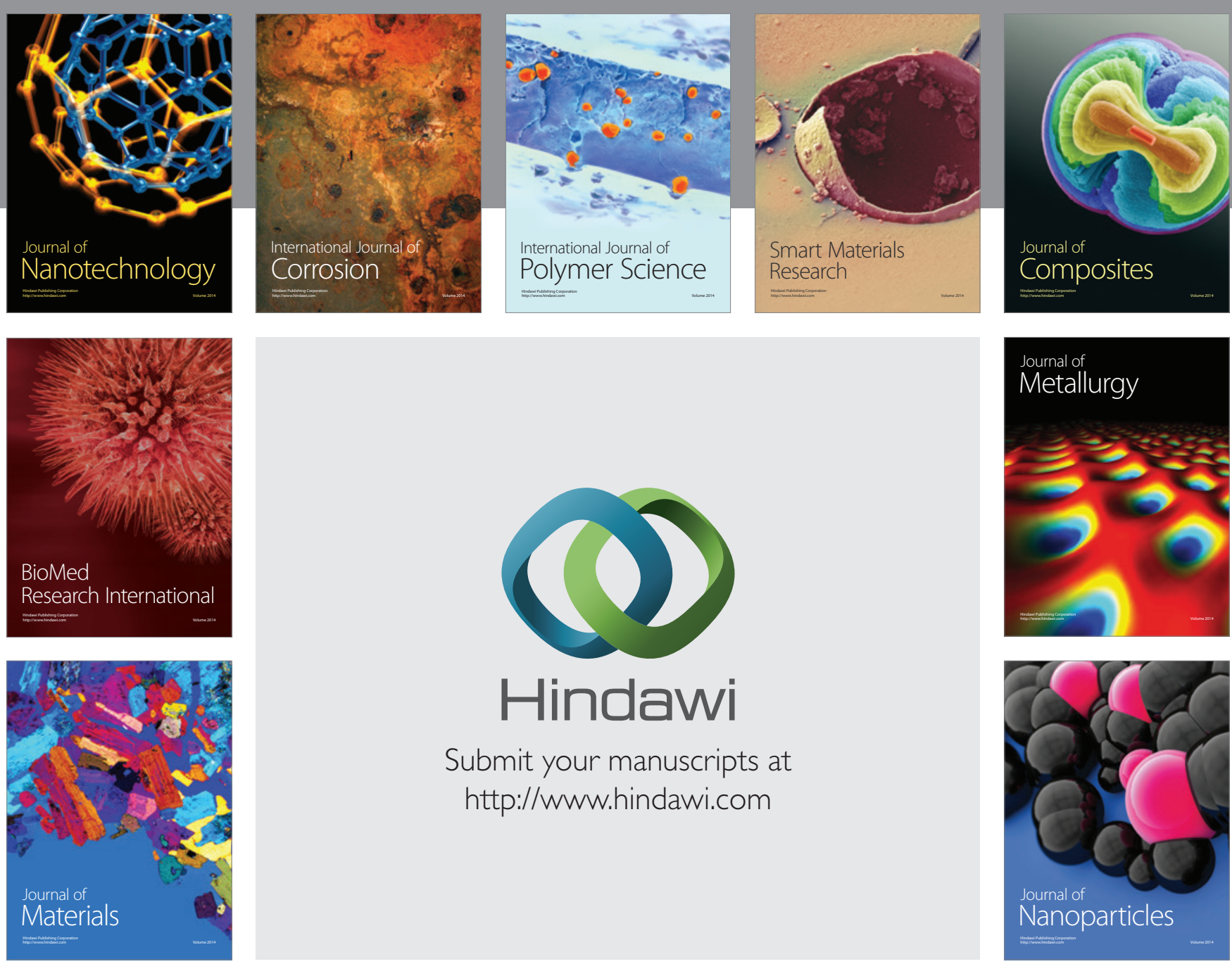

Submit your manuscripts at http://www.hindawi.com
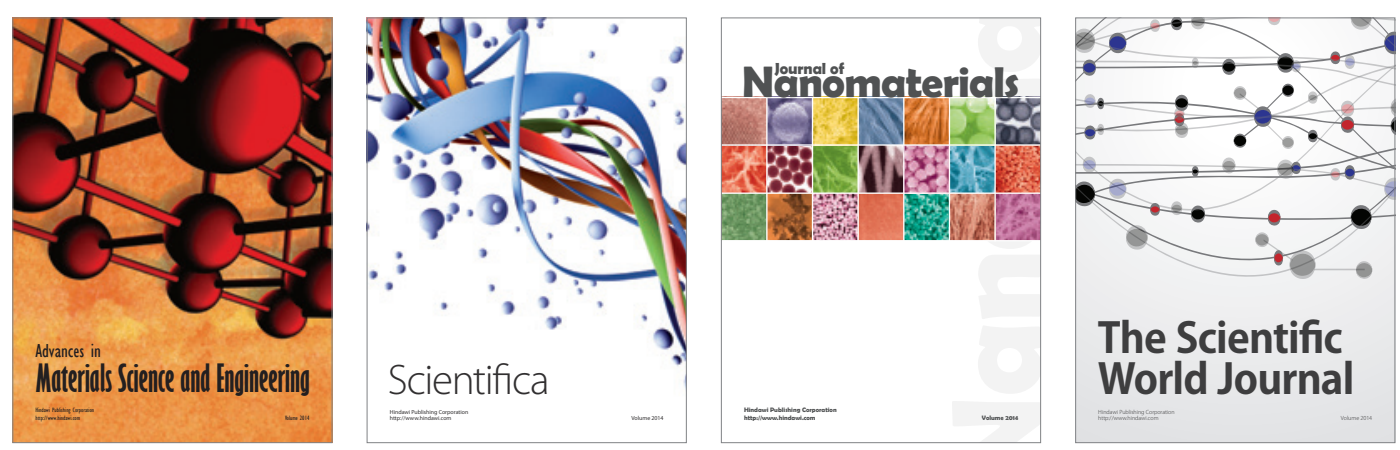

\section{The Scientific World Journal}
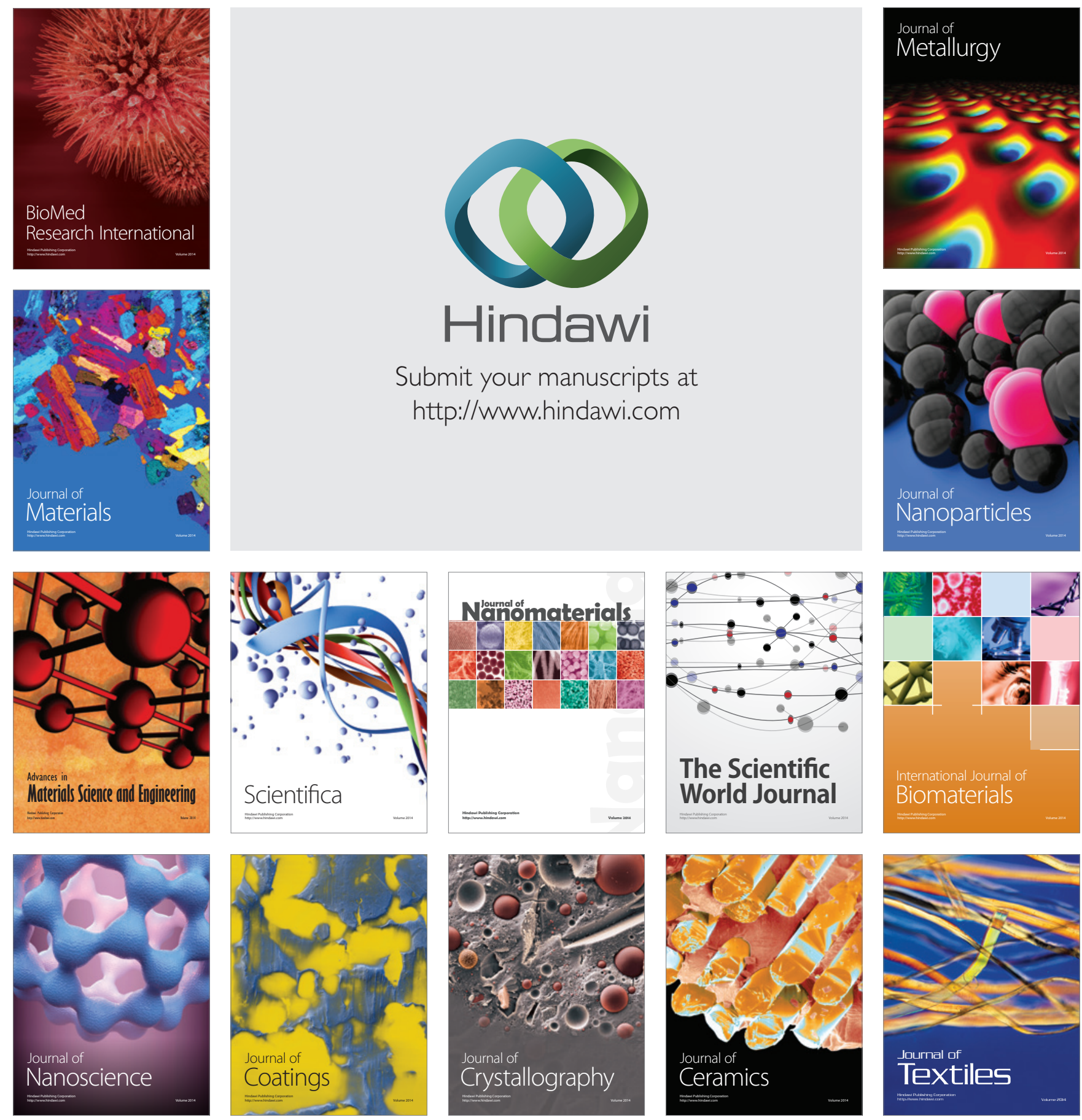